\title{
Exciton formation in dye doped OLEDs using electrically detected magnetic resonance
}

\author{
Augusto Batagin-Neto ${ }^{a}$, Jorge A. Gómez ${ }^{b}$, Fernando A. Castro ${ }^{c}$, Frank Nüesch ${ }^{d}$, Libero Zuppirolie, \\ Carlos F. O. Graeff ${ }^{f}$ \\ a UNESP - Univ Estadual Paulista, POSMAT - Prog de Pós-Grad em Ciência e Tecnologia de \\ Materiais, Bauru, SP, Brazil; \\ b Departamento de Física, FFCLRP-USP, Ribeirão Preto, São Paulo, Brazil; \\ ${ }^{c}$ Materials Division, National Physical Laboratory, Teddington, UK; \\ ${ }^{d}$ Empa, Swiss Federal Laboratories for Materials Testing and Research, Laboratory for Functional \\ Polymers, Dübendorf, Switzerland; \\ e Laboratoire d'optoélectronique dês matériaux moléculaire, Lausanne, Switzerland \\ ${ }^{\mathrm{f}}$ Departamento de Física, FC-UNESP, Bauru, São Paulo, Brazil;
}

\begin{abstract}
Electrically Detected Magnetic Resonance (EDMR) was used to investigate the influence of dye doping molecules on spin-dependent exciton formation in Aluminum (III) 8-hydroxyquinoline ( $\left.\mathrm{Alq}_{3}\right)$ based OLEDs with different device structures and temperature ranges. 4-(dicyanomethylene)-2-methyl-6-\{2-[(4-diphenylamino-phenyl]ethyl $\}-4 \mathrm{H}-$ pyran (DCM-TPA) and 5,6,11,12-tetraphenylnaphthacene (Rubrene) were used as dopants. A strong temperature dependence have been observed for doped OLEDs, with a decrease of two orders of magnitude in EDMR signal for temperatures above $\sim 200 \mathrm{~K}$. The signal temperature dependence were fitted supposing different spin-lattice relaxation processes. The results suggest that thermally activated vibrations of dopants molecules induce spin pair dissociation, reducing the signal.
\end{abstract}

Keywords: $\mathrm{Alq}_{3}$; dye doped OLEDs; DCM-TPA; Rubrene; EDMR; spin-relaxation processes.

\section{INTRODUCTION}

Organic light emitting diodes (OLEDs) have been rapidly inserted into the market and applications demand due to the color tunability as well as color purity and color saturation of these devices.

In particular, aluminum (III) 8-hydroxyquinoline ( $\left.\mathrm{Alq}_{3}\right)$ has been extensively used as light emitting-electron transporting layer in OLEDs. The first report of a high-performance Alq3-based organic eletroluminescent (EL) device presented a reasonable external quantum efficiency ${ }^{1}$, despite its low fluorescence efficiency. It is known that OLEDs EL quantum yield is limited, in part, by fluorescence quantum efficiency of the emitter material, in this sense, nowadays high efficiency OLEDs make use of highly fluorescent dyes introduced into the emissive layer (typically by co-evaporation) for EL quantum yield improvement ${ }^{2}$. As a matter of fact, dye doping process leads to a series of advantages relative to undoped devices, such as significantly narrower EL spectra ${ }^{3}$ and much higher EL efficiency. ${ }^{4}$

However, despite the increasing use of dye doped OLEDs, there is not a satisfactory knowledge about the dominant recombination mechanisms in these devices. In particular, the discussion is centered on the yield of triplet-to-singlet exciton formation ${ }^{5}$ and on the possible processes, energy ${ }^{6}$ or charge transfer ${ }^{7}$, that increases device efficiency. In fact, just a few of experimental techniques can address these questions, under real operating conditions. In this sense the use of techniques such as the Electrically Detected Magnetic Resonance (EDMR) has been shown to be relevant ${ }^{8}$.

The EDMR technique consists basically in a measurement of the device current changes under magnetic resonance conditions. It permits to relate the properties of electric transport with the wave functions of the participating molecules, and it has been widely used in the study of transport properties of organic and inorganic devices $9,10,8$.

Photonics and Optoelectronics Meetings (POEM) 2011: Optoelectronic Devices and Integration, edited by

Erich Kasper, Jinzhong Yu, Xun Li, Xinliang Zhang, Jinsong Xia, Junhao Chu, Zhijiang Dong, Bin Hu, Yan Shen,

Proc. of SPIE Vol. 8333, 83331B - @ 2012 SPIE · CCC code: 0277-786X/12/\$18 - doi: 10.1117/12.918246

Proc. of SPIE Vol. 8333 83331B-1 
Recently undoped and dye doped $\mathrm{Alq}_{3}$-based devices have been studied using EDMR ${ }^{10,8}$. In these works this technique has demonstrated high sensitivity and selectivity to different spin systems formed in the conduction process. In special, is has shown to be a interesting tool to probe the spin states just before exciton formation, i.e. the precursor pair formed by cationic (hole) and anionic (electron) molecular species with possible different spin states ${ }^{8}$.

In this paper, we used EDMR to investigate the influence of dye doping on spin dependent exciton formation in $\mathrm{Alq}_{3}-$ based OLEDs. We measured the EDMR spectra at different temperatures and performed a phase analysis of the EDMR signals to observe the spectral characteristics of each spin system that forms the exciton. The results reinforce the known fact that the recombination zone of the electron-hole pair in OLEDs is located near the $\alpha$-NPD layer ${ }^{11}$. A strong temperature dependence of EDMR signal was observed for doped structures. Considering the spin-dependent behavior of conductivity with the spin-spin relaxation time limited by $\mathrm{T}_{1}$ (or spin coherence time) ${ }^{12}$, the temperature dependence were fitted supposing different spin-lattice relaxation processes ${ }^{13}$.

\section{METHODOLOGY}

Figure 1 presents the devices structures studied and the frontier molecular orbitals energies of each compound (HOMO: highest occupied molecular orbital and LUMO: lowest unoccupied molecular orbital). Alq 3 has been used as emitter material and 4-(dicyanomethylene)-2-methyl-6-\{2-[(4-diphenylamino)-phenyl]ethyl $\}-4 \mathrm{H}-$ pyran (DCM-TPA) and $5,6,11,12$-tetraphenyl naphthacene (Rubrene) have been used as dopants.

The devices were manufactured by thermal evaporation on glass substrates under high vacuum. An active area of $2 \times 2$ $\mathrm{mm}^{2}$ have been used. Indium-tin-oxide (ITO) coated glass substrates were cleaned in a sequence of ultrasonic baths using ethanol, acetone, detergent and Milli-Q water, respectively. The organic layers were deposited in sequence.

For the undoped OLED (Fig. 1), used as a reference, the sequence comprises a $10 \mathrm{~nm}$ thick copper phthalocyanine (CuPc) layer, used to improve hole injection, followed by a $40 \mathrm{~nm}$ thick $\mathrm{N}, \mathrm{N}^{\prime}$-diphenyl-N, $\mathrm{N}^{\prime}$-bis(1-naphthyl)-1,1 'biphenyl-4,4" diamine ( $\alpha-\mathrm{NPD}$ ) hole transporting layer and a $60 \mathrm{~nm}$ thick $\mathrm{Alq}_{3}$ electron transporting and emissive layer. Subsequently, a $0.8 \mathrm{~nm}$ thick LiF layer was deposited followed by a $100 \mathrm{~nm}$ thick Al layer. The DCM-TPA and Rubrenea dye doped devices were prepared in the same way, except that instead of the $60 \mathrm{~nm} \mathrm{Alq} 3$ layer, a $20 \mathrm{~nm}$ thick Alq 3 doped layer (co-evaporation of $\mathrm{Alq}_{3}$ with $1 \mathrm{wt} . \%$ of dye doping molecules) was deposited followed by a $40 \mathrm{~nm}$ thick undoped $\mathrm{Alq}_{3}$ layer. For the Rubrene-b OLED, $20 \mathrm{~nm}$ of $\mathrm{Alq}_{3}$ was deposited, followed by a $20 \mathrm{~nm}$ thick Alq $\mathrm{Al}_{3}$ doped layer (co-evaporation of Alq 3 with $1 \mathrm{wt} .-\%$ of Rubrene), followed by an extra layer of $20 \mathrm{~nm}$ of $\mathrm{Alq}_{3}$.

In order to avoid air-induced degradation, the devices were directly transferred to an inert gas glove box without atmosphere exposure. Samples were then contacted and sealed inside an ESR quartz tube.

EDMR measurements were done using a specially designed computer interfaced K-Band (24 GHz) electron spin resonance (ESR) spectrometer, in the temperature range from 100 to $300 \mathrm{~K}$. Spin-dependent conductivity changes were measured by modulating the static magnetic field $\mathrm{H}_{0}$ and using lock-in detection of the current changes. The magneticfield modulation used was $133 \mathrm{~Hz}$. EDMR signal amplitude $(\Delta \sigma / \sigma)$ was calculated from the current changes: $\left(\mathrm{I}-\mathrm{I}_{0}\right) / \mathrm{I}_{0}$, where $\mathrm{I}_{0}$ is the current out of resonance. All measurements were realized in the OLED electroluminescent regime.

The regressions fits for EDMR signals temperature dependence were performed with QtiPlot software package. ${ }^{14}$

\section{RESULTS AND DISCUSSIONS}

The devices structures presented in Fig. 1 were specially designed to study the mechanism associated to the origin of light emission in dye doped OLEDs. 


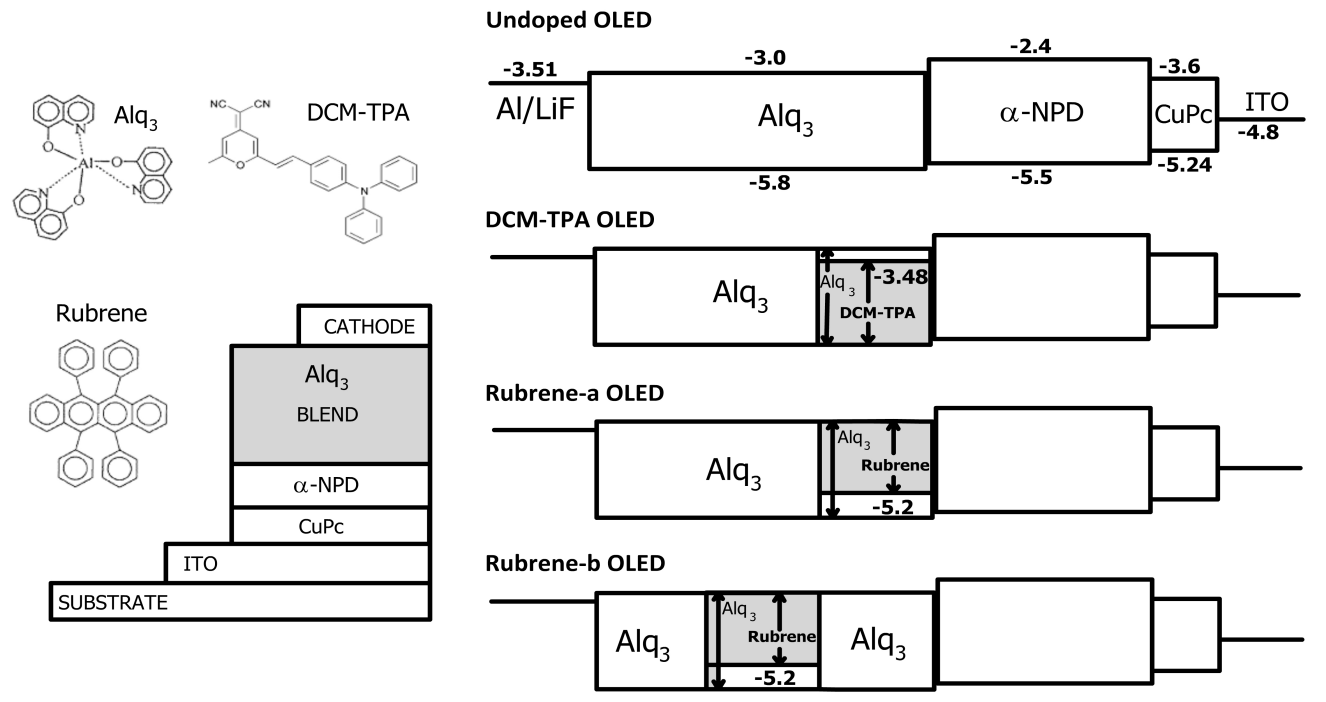

Figure 1. OLEDs devices structure and energy levels of their components (eV): i) Undoped Alq ${ }_{3}$, ii) DCM-TPA dye doped, iii) Rubrene dye doped, and iv) Rubrene dye doped OLED with an extra $\mathrm{Alq}_{3}$ layer between the doped $\mathrm{Alq}_{3}$ and the $\alpha$-NPD.

Figure 2 shows the typical EDMR signal detected for Rubrene-b OLED at two bias currents at the three phases: $0^{\circ}, 89.8^{\circ}$ and $90.2^{\circ}$. Two different signals can be uncoupled as discussed elsewhere ${ }^{8}$ what have been associated with anionic (electron-e) and cationic (hole-h) molecular species.

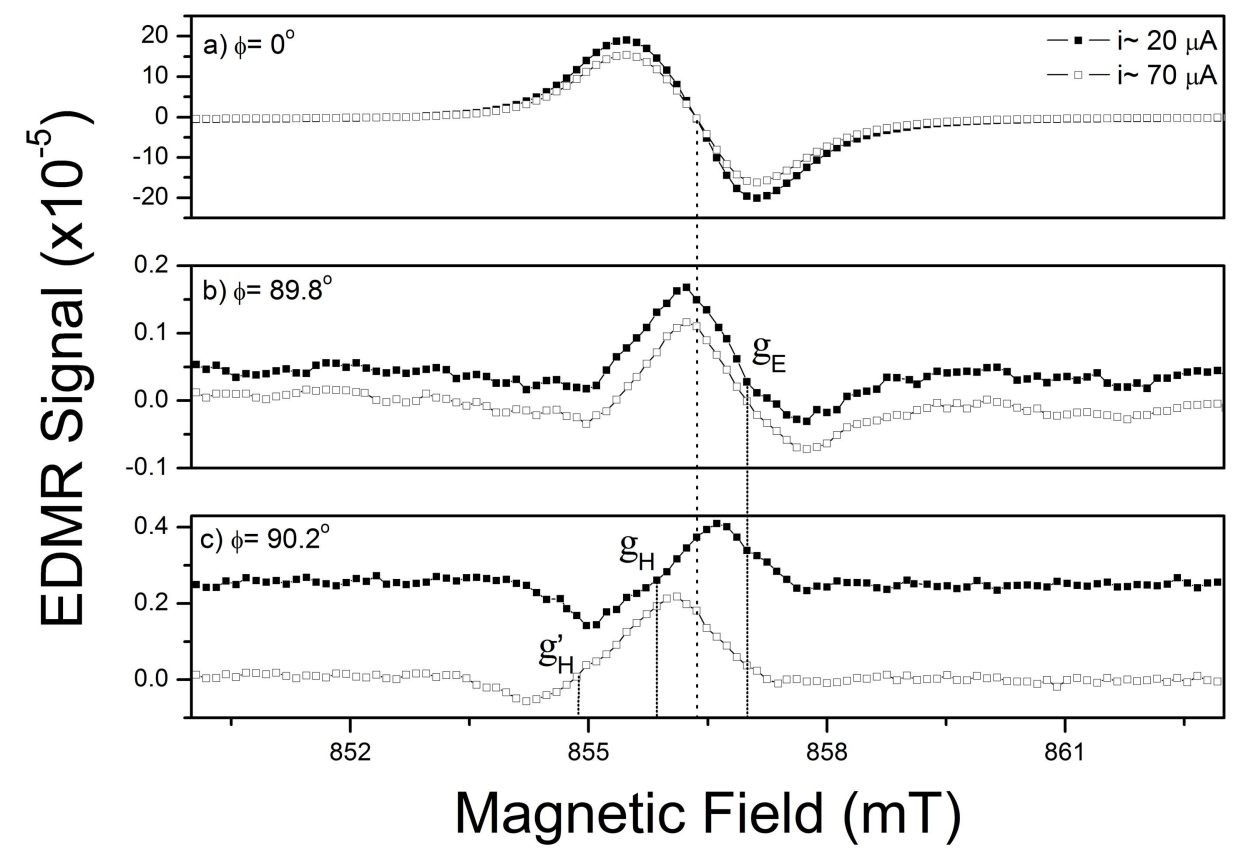

Figure 2. Typical EDMR signal of the undoped $\mathrm{Alq}_{3}$ OLED showed in Fig. 1. The EDMR signals for three different phases are shown: a) $\varphi=0^{\circ}$, b) $\varphi=89.8^{\circ}$ and c) $\varphi=90.2^{\circ}$.

As can be seen, two different $\mathrm{g}$-factors can be resolved for phases close to $90^{\circ}: \mathrm{g}_{\mathrm{E}}=2.0028 \pm 0.0003$ and $\mathrm{g}_{\mathrm{H}}=2.004 \pm$ 0.0002 at $\sim 20 \mu \mathrm{A}$ bias current. At bias current of $\sim 70 \mu \mathrm{A}$, the g-factor $\mathrm{g}_{\mathrm{H}}$ have shown a shift to $\mathrm{g}_{\mathrm{H}}=2.005 \pm 0.0003$. Table 1 summarizes the EDMR spectral characteristics for each device architecture. 
Table 1. Spectral characteristics of the EDMR signals components for the different devices (at $100 \mathrm{~K}$ ).

\begin{tabular}{|lcccc|}
\hline \multicolumn{1}{|c}{ Device } & $\mathbf{g}_{\mathbf{H}}$ & $\begin{array}{c}\mathbf{\Delta} \mathbf{h p \mathbf { H } ^ { \mathrm { H } }} \\
\mathbf{( \mathbf { m T } )}\end{array}$ & $\mathbf{g}_{\mathbf{E}}$ & $\begin{array}{c}\Delta \mathbf{H p \mathbf { H } ^ { \mathrm { E } }} \\
\mathbf{( \mathbf { m T } )}\end{array}$ \\
\hline Undoped $\left(\mathrm{I}_{0} \sim 20-100 \mu \mathrm{A}\right)$ & $2.0040 \pm 0.0001$ & 1.50 & $2.0028 \pm 0.0002$ & $2.0-3.4$ \\
DCM-TPA $\left(\mathrm{I}_{0} \sim 20 \mu \mathrm{A}\right)$ & $2.0040 \pm 0.0002$ & 1.82 & $\mathbf{2 . 0 0 3 9} \pm \mathbf{0 . 0 0 0 1}$ & $3.0-4.2$ \\
Rubrene-a $\left(\mathrm{I}_{0} \sim 20 \mu \mathrm{A}\right)$ & $\mathbf{2 . 0 0 5 1} \pm \mathbf{0 . 0 0 0 2}$ & 1.69 & $2.0028 \pm 0.0002$ & $2.2-3.1$ \\
Rubrene-b $\left(\mathrm{I}_{0} \sim 20 \mu \mathrm{A}\right)$ & $2.0040 \pm 0.0002$ & 1.53 & $2.0028 \pm 0.0003$ & $2.0-3.1$ \\
Rubrene-b $\left(\mathrm{I}_{0}>50 \mu \mathrm{A}\right)$ & $\mathbf{2 . 0 0 5 0} \pm \mathbf{0 . 0 0 0 3}$ & 1.70 & $2.0028 \pm 0.0003$ & $2.1-3.1$ \\
\hline
\end{tabular}

The data presented in Table 1 suggest that the inclusion of dopants into $\mathrm{Alq}_{3}$ layer induces differences in the g-factor values, $\mathrm{g}_{\mathrm{H}}$ and $\mathrm{g}_{\mathrm{E}}$. These dopants may act as trapping states for holes (Rubrene) or for electrons (DCM-TPA), changing the environment of charge carriers in the resonance condition. In fact the g-factor of the cationic states for the devices without hole traps (undoped and DCM-TPA device) is different from the g-factor value of the Rubrene-a. Also, the gfactor value of the anionic states for the undoped and Rubrene-a devices is different from the g-factor value of the DCMTPA device, what suggests the formation of different precursor species in these devices. This hypothesis is reinforced by Rubrene-b device, which presents similar spectral characteristics of undoped one. Since the main recombination zone is near the $\alpha$-NPD layer, ${ }^{11}$ no change was expected for Rubrene-b device (at least for low currents), what is observed in Table 1. However, at higher bias currents, the space charge accumulation becomes more important at the doped/undoped emissive interface leading to a shift of the recombination zone towards the ITO side. ${ }^{15}$ These results indicate that dopants acts as charge traps; so that the exciton formation is performed predominantly by a cation from Rubrene and an anion from $\mathrm{Alq}_{3}$, for Rubrene doped OLEDS; and by an anion from DCM-TPA and a cation from $\mathrm{Alq}_{3}$, for DCM-TPA doped OLEDS.

Figure 3 shows the temperature dependence of the normalized EDMR signals amplitudes $(\Delta \sigma / \sigma)$ for a phase signal of $\phi=$ $0^{\circ}$. The inset shows the EDMR spectra of device Rubrene-a structure, at 300 and $100 \mathrm{~K}$. All measurements were obtained for devices operating at the same bias current $(\sim 20 \mu \mathrm{A})$.

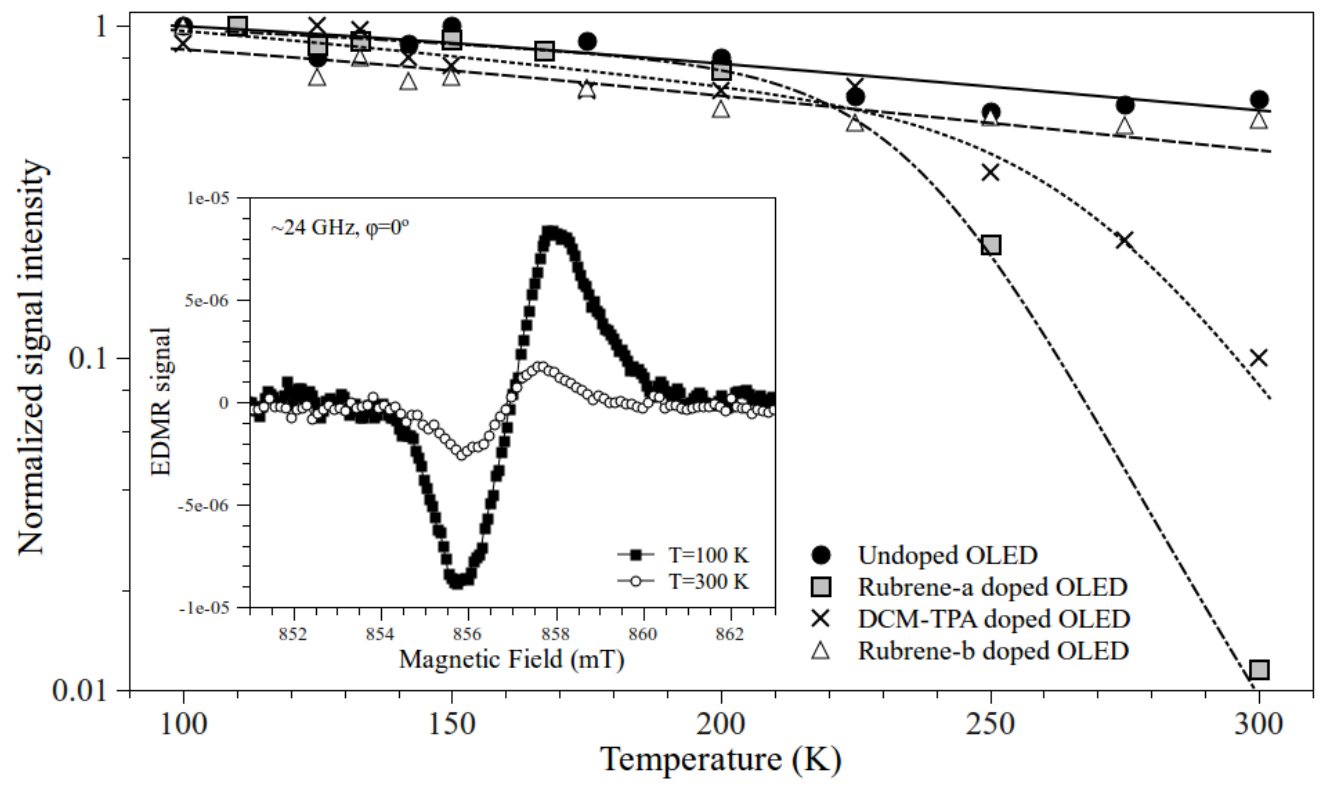

Figure 3. Normalized EDMR signals amplitudes $(\Delta \sigma / \sigma)$ as a function of temperature for the different OLEDs: experimental points and adjusted curves using Eq. 2 and 3 with the parameters presented in Table 2. The graphic inset show EDMR signals $\left(\varphi=0^{\circ}\right)$ of the Rubrene dye doped OLEDs without extra $\mathrm{Alq}_{3}$ layer at two different temperatures. 
As can be seen, the temperature dependence of the signal for DCM-TPA and Rubrene-a OLEDs is different from that of the undoped and Rubrene-b OLEDs (at low bias). DCM-TPA and Rubrene-a doped structures show strong temperature dependence with a decrease of two orders in the magnitude of the EDMR signal at temperatures above 225 and $200 \mathrm{~K}$, respectively. On the other hand, a weak dependence have been observed in undoped and Rubrene-b OLEDs. These results reinforce the known fact that the recombination zone of the electron-hole pair in OLEDs is located near the $\alpha$ NPD layer and that the signal temperature dependence is due to dopants molecules.

Recently, the temperature dependence in DCM-TPA doped devices has been attributed to a decrease in spin coherence time or change in exciton precursor reaction rates ${ }^{16}$. Supposing a homogeneously broadened Lorentzian line and assuming the spin-spin relaxation time limited by the spin-lattice relaxation time, such as $\mathrm{T} 1 \approx \mathrm{T} 2,{ }^{12}$ the EDMR signal can be described by:

$$
\begin{aligned}
& \frac{\Delta \sigma}{\sigma}=K \cdot \frac{\omega_{1}^{2} T_{1}^{2}}{\omega_{1}^{2} T_{1}^{2}+1} \\
& \frac{\Delta \sigma}{\sigma}=\frac{K}{1+\left(\frac{1}{\omega_{1} T_{1}}\right)^{2}}
\end{aligned}
$$

since $\Delta \sigma / \sigma$ represents the signal intensity; $\omega_{1}$ represents the microwave-induced spin-flip rate (proportional to the microwave power); $\mathrm{T}_{1}$ represents the spin-lattice relaxation time and $\mathrm{K}$ is a normalization constant (note that polarization effects have not been considered).

In order to evaluate the influence of temperature in the signal, the temperature dependence of $T_{1}$ were fitted supposing different spin-lattice relaxation processes. ${ }^{13}$

$$
\frac{1}{\omega_{1} T_{1}}=A_{d i r} T+A_{\exp } e^{\frac{-\Delta}{T}}
$$

the first component in the right side represents the direct relaxation process and the second component represents a exponential decay term, compatible with several different relaxation processes (Orbach, Local Vibration Mode and Thermally Actived). ${ }^{13}$ The addition of a Raman relaxation component in Eq. 3 have not shown significant improvement in the fitted curves adjustment and were not shown. Table 2 presents the regressions parameters for the best fitted curves shown in the Fig. 3.

Table 2. Parameters employed for fit of the temperature dependence of EDMR signal shown in Fig. 3.

\begin{tabular}{|ccccc|}
\hline Parameters & $\begin{array}{c}\text { Undoped } \\
\text { OLED }\end{array}$ & $\begin{array}{c}\text { DCM-TPA Doped } \\
\text { OLED }\end{array}$ & $\begin{array}{c}\text { Rubrene-a Doped } \\
\text { OLED }\end{array}$ & $\begin{array}{c}\text { Rubrene-b Doped } \\
\text { OLED }\end{array}$ \\
\hline $\mathrm{A}_{\mathrm{dir}}$ & 0.0034 & 0.0042 & 0.0030 & 0.0038 \\
$\mathrm{~A}_{\exp }$ & --- & $0.92 \times 10^{5}$ & $2.06 \times 10^{5}$ & --- \\
$\Delta$ & --- & 3180.47 & 2995.41 & --- \\
$\mathrm{K}$ & 1.10 & 1.13 & 1.06 & 0.97 \\
\hline
\end{tabular}

Note that the temperature dependence in undoped and Rubrene-b OLEDs structures have been fitted only considering direct relaxation process components. On the other hand, DCM-TPA and Rubrene-a structures presents an exponential decay component, with an activation energy $\left(\mathrm{E}_{\mathrm{a}}\right)$ of 0.274 and $0.258 \mathrm{eV}$, respectively.

The activation energies obtained for these process is compatible with structural transitions in dopants molecules. In fact, both dopants presents two stable isomer conformation as shown in Figure 4. Rubrene presents planar and twisted isomers with a transition energy of about $0.120 \mathrm{eV} .{ }^{17}$ The most stable structure is the twisted one, but changes in the population equilibrium is expected under heating, promoting the thermochromic effects observed in this compound. ${ }^{18}$ In addition, it is known that Rubrene presents a temperature dependent intersystem crossing and triplet-triplet absorption in solid solution with activation energy between 0.107 and $0.570 \mathrm{eV},{ }^{19}$ which is compatible with a relaxation process 
involving an intermediate upper level as Orbach process. On the other hand, DCM-TPA compound presents a triphenylamine group with a three-bladed propeller structure and a two mirror-like potential energy minima with a transition energy of about $0.207 \mathrm{eV} .{ }^{20}$

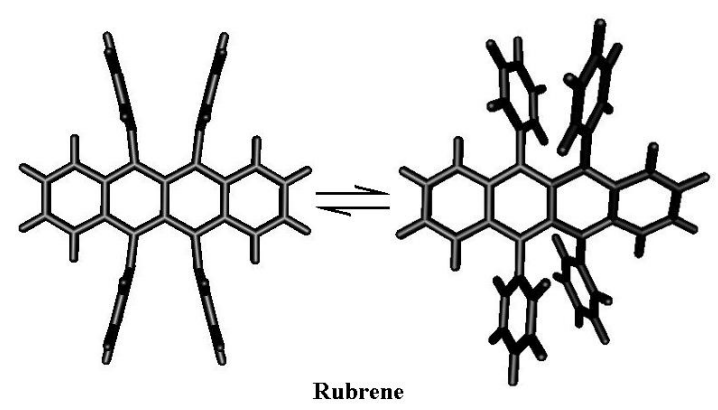

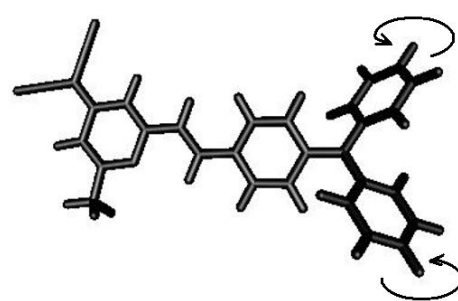

DCM-TPA

Figure 4. Structure of Rubrene and DCM-TPA isomers.

According with Kaplan-Solomon-Mott mechanism, the EDMR signal can be attributed to precursor pairs formed before the spin-dependent transition ${ }^{21}$. If the spin configuration of these bounded pair is singlet the transition occurs (hopping or recombination) with a characteristic time constant. On the other hand, if the transition is spin-forbidden, it will occur only if one of the two spins flips, or by spontaneous spin-lattice relaxation processes (with a time constant $T_{1}$ ), or due to microwave-induced process (with a time constant $\tau_{\mathrm{mw}}$ ). In addition, if the transition takes longer time to occur, the bounded pair can also be dissociate with a time constant $\tau_{\mathrm{d}}{ }^{22}$, thus $T_{1}$ can be dominated by $\tau_{\mathrm{d}}$.

Our results suggest a influence of lattice vibrations in the precursor pair dissociation time. In organic molecular materials, the precursor pairs are expected to form between charged species (cationic and anionic molecules) from charge carriers hopping; these excited pairs are then kept bounded by electrostatic interactions, similarly to electromers. Our results indicate an exponential decay of $T_{1}$ with energies activation compatible with structural transitions of dopants structures. These thermally activated transitions could promote small changes in the intermolecular distances of cationic/anionic species and facilitate the spin pair dissociation, reducing the EDMR signal.

\section{CONCLUSIONS}

Electrically Detected Magnetic Resonance (EDMR) was used to investigate the influence of dye doping molecules on spin-dependent exciton formation in Aluminum (III) 8-hydroxyquinoline (Alq3) based OLEDs with different device structures and temperature ranges. The results suggest that dopants molecules act as trapping states for holes (Rubrene) or for electrons (DCM-TPA), such as exciton formation is performed predominantly by a cation from Rubrene and an

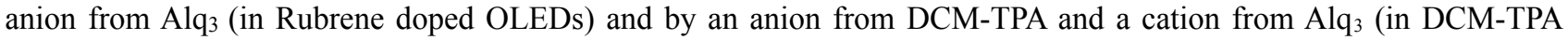
doped OLEDs). In addition, doped dye OLEDs presents a strong temperature dependence. The dependence in undoped and Rubrene-b structures have been fitted considering only a direct relaxation process. On the other hand, DCM-TPA and Rubrene-a structures presents an exponential decay component compatible with structural transitions of dopants molecules. These results suggest a influence of lattice vibrations in the precursor pair dissociation time, a subject still barely discussed in the literature.

\section{ACKNOWLEDGMENTS}

The authors would like to thank Dr. Douglas M. G. Leite for fruitful discussions . This research was sponsored by the following agencies: FAPESP, CLAF/CNPq, CAPES and INCTMN-FAPESP/CNPq.

\section{REFERENCES}

[1] C. W. Tang and S. A. VanSlyke, "Organic electroluminescent diodes," Applied Physics Letters 51, 913-915 (1987).

[2] C. W. Tang, S. A. VanSlyke, and C. H. Chen, "Electroluminescence of doped organic thin films," Journal of Applied Physics 65, 3610-3616 (1989).

[3] A. A. Shoustikov, Yujian You, and M. E. Thompson, "Electroluminescence color tuning by dye doping in organic light-emitting diodes," IEEE Journal of Selected Topics in Quantum Electronics 4, 3-13 (1998). 
[4] J. Kido and Y. Iizumi, "Fabrication of highly efficient organic electroluminescent devices," Applied Physics Letters 73, 2721-2723 (1998).

[5] M. Segal, M. Baldo, R. Holmes, S. Forrest, and Z. Soos, "Excitonic singlet-triplet ratios in molecular and polymeric organic materials," Physical Review B 68, 075211 (2003).

[6] R. N. Bera, Y. Sakakibara, M. Tokumoto, and K. Yase, "Time-Resolved Photoluminescence Study on Energy Transfer from Alq3 (tris(8-hydroxyquinoline)aluminum) to Red-Emissive Tetraphenylchlorin,” Japanese Journal of Applied Physics 42, 7379-7380 (2003).

[7] X. Gong, J. C. Ostrowski, D. Moses, G. C. Bazan, and A. J. Heeger, "Electrophosphorescence from a Polymer Guest-Host System with an Iridium Complex as Guest: Förster Energy Transfer and Charge Trapping," Advanced Functional Materials 13, 439-444 (2003).

[8] C. F. O. Graeff, G. B. Silva, F. Nüesch, and L. Zuppiroli, "Transport and recombination in organic light-emitting diodes studied by electrically detected magnetic resonance," The European Physical Journal E 18, 21-28 (2005).

[9] C. Graeff, M. Brandt, M. Stutzmann, M. Holzmann, G. Abstreiter, and F. Schäffler, "Electrically detected magnetic resonance of two-dimensional electron gases in Si/SiGe heterostructures," Physical Review B 59, 13242-13250 (1999).

[10] F. Castro, "Electrically detected magnetic resonance of organic and polymeric light emitting diodes," Journal of Non-Crystalline Solids 338-340, 622-625 (2004).

[11] Y.-Q. Peng, Q.-S. Yang, H.-W. Xing, X.-S. Li, J.-T. Yuan, C.-Z. Ma, and R.-S. Wang, "Recombination zone and efficiency in bipolar single layer light-emitting devices: a numerical study," Applied Physics A 93, 559-564 (2008).

[12] C. Graeff, M. Stutzmann, and M. Brandt, "Spin-dependent photoconductivity in hydrogenated amorphous germanium and silicon-germanium alloys," Physical Review B 49, 11028-11034 (1994).

[13] Y. Zhou, B. E. Bowler, G. R. Eaton, and S. S. Eaton, "Electron spin lattice relaxation rates for S=12 molecular species in glassy matrices or magnetically dilute solids at temperatures between 10 and $300 \mathrm{~K}$," J. Magn. Reson. 139, 165-174 (1999).

[14] I. Vasilef, QtiPlot: data analysis and scientific visualization.

[15] D. Berner, "Splitting of the recombination zone in organic light emitting diodes by dye doping," Journal of Applied Physics 95, 3749-3754 (2004).

[16] W. Bin, L. Ying-Jie, L. Ji-Zhong, L. Lin, C. Jin, W. Jun, and Z. Jian-Hua, "Dependence of charge trapping of fluorescent and phosphorescent dopants in organic light-emitting diodes on the dye species and current density," Chinese Physics B 19, 037105 (2010).

[17] M. Kytka, L. Gisslen, A. Gerlach, U. Heinemeyer, J. Kováč, R. Scholz, and F. Schreiber, "Optical spectra obtained from amorphous films of rubrene: Evidence for predominance of twisted isomer," The Journal of Chemical Physics 130, 214507 (2009).

[18] A. Schonberg, A. Mustafa, and W. Asker, Journal of the American Chemical Society 76, 4134-4136 (1954).

[19] L. Song, "Temperature dependent intersystem crossing and triplet-triplet absorption of rubrene in solid solution," Journal of Luminescence 50, 75-81 (1991).

[20] I. Reva, L. Lapinski, N. Chattopadhyay, and R. Fausto, "Vibrational spectrum and molecular structure of triphenylamine monomer: A combined matrix-isolation FTIR and theoretical study," Physical Chemistry Chemical Physics 5, 3844-3850 (2003).

[21] D. Kaplan, I. Solomon, and N. F. Mott, "Explanation of the large spin-dependent recombination effect in semiconductors," Journal de Physique Lettres 39, 51-54 (1978).

[22] M. Stutzmann, M. S. Brandt, and M. W. Bayerl, "Spin-dependent processes in amorphous and microcrystalline silicon: a survey,” Journal of Non-Crystalline Solids 266-269, 1-22 (2000). 